\title{
Modelos matemáticos na predição do comportamento higroscópico para pó do mix de batata yacon e suco de lima
}

\section{Application of mathematical models in the prediction of the hygroscopic dehavior of the potato mix yacon powder and lemon juice}

\author{
Regilane Marques Feitosa ${ }^{1 *}$; Renato Costa da Silva ${ }^{2}$; Lumara Tatiely Santos Amadeu ${ }^{3}$; Rossana Maria Feitosa Figueiredo ${ }^{4}$ \\ Alexandre José Melo Queiroz ; Emanuel Neto Alves de Oliveira ${ }^{5}$
}

Resumo: A batata yacon tornou-se popular devido aos inúmeros benefícios que proporciona à saúde, porém seu tempo de vida útil é curto, aliado ao escurecimento enzimático, comum em alimentos ricos em compostos fenólicos, que reduz e restringe o seu consumo. Em virtude de ser um produto de alto valor, e com o intuito de se evitar o seu desperdício, justifica-se o estudo de meios de conservação do mesmo. A secagem seguida por transformação do produto em pó se apresenta como uma alternativa conveniente, segura e capaz de proporcionar longa vida útil ao material processado. Diante do exposto, objetivou-se verificar o comportamento higroscópico do pó obtido pela liofilização do mix de batata yacon e suco de lima ácida Tahiti nas temperaturas de 20,30 e $40{ }^{\circ} \mathrm{C}$. Elaborou-se um mix de batata yacon com suco de lima ácida Tahiti, aproveitando a propriedade deste suco de atuar na inativação enzimática da enzima polifenoloxidase, além de ser fonte de vitamina C. O mix com maltodextrina foi seco por liofilização e o pó resultante foi submetido à análise de comportamento higroscópico, determinando-se as isotermas de adsorção de umidade nas temperaturas de 20, 30 e $40{ }^{\circ} \mathrm{C}$ e ajustando-se os modelos matemáticos de Peleg, GAB, Halsey, Oswin e Smith às isotermas. O modelo de Peleg foi o que melhor se ajustou às isotermas de adsorção de umidade do pó da batata yacon com suco de lima ácida Tahiti. As isotermas foram classificadas como Tipo II e a umidade na monocamada molecular apresentou valor em torno de $7 \%$ b.s.

Palavras-chave: Atividade de água; Isotermas de adsorção; Smallanthus sonchifolia

\begin{abstract}
The yacon potato became popular because of the numerous benefits it provides to health, but their life span is short, combined with enzymatic browning, common in foods rich in phenolic compounds, which reduces and limits its consumption. By virtue of being a product of high value and in order to avoid wastage, justified the study of conservation means where drying followed by processing of the product powder is presented as a convenient alternative, safe and able to provide long useful life to processed material. Given the above, it elaborated a yacon potato mix with lemon juice, taking ownership of this juice to act in the enzymatic inactivation of PPO, besides being a source of vitamin $\mathrm{C}$. The mix with maltodextrin was then dried by lyophilisation and resulting powder was subjected to the hygroscopic behavior analysis, determining the moisture adsorption isotherms at temperatures of 20,30 and $40{ }^{\circ} \mathrm{C}$, and adjusting the mathematical models Peleg, GAB, Halsey, Oswin and Smith isotherms. The Peleg model was the best fit the moisture adsorption isotherms of powder yacon potato with lemon juice; isotherms were classified as Type II and moisture in the molecular monolayer presented value around $7 \%$ b.s.
\end{abstract}

Key words: Water activity; Adsorption isotherm; Smallanthus sonchifolius.

\footnotetext{
*Autor para correspondência

Recebido para publicação em 09/03/2017; aprovado em 25/06/2017

${ }^{1}$ Pesquisadora PNPD/CAPES, Engenharia de Processos, Universidade Federal de Campina Grande, Campina Grande -PB, regilanemarques@ gmail.com.

${ }^{2}$ Mestrando em Engenharia Agrícola, Universidade Federal de Campina Grande, Campina Grande -PB, renatinocosta@gmail.com

${ }^{3}$ Graduanda em Engenharia Agrícola, Universidade Federal de Campina Grande, Campina Grande -PB, lumaratatiely@hotmail.com

${ }^{4}$ Docente/pesquisador do Departamento de Engenharia agrícola, Universidade Federal de Campina Grande, Campina Grande -PB, rossana@deag.ufcg.edu.br, alex@deag.ufcg.edu.br

${ }^{5}$ Docente do Instituto Federal de Educação, Ciência e Tecnologia do Rio Grande do Norte, Pau dos Ferros - RN, emanuel.oliveira16@ gmail.com
} 


\section{INTRODUÇÃO}

A batata yacon é um tubérculo composto principalmente por água e pelos carboidratos frutose, glicose, sacarose, frutooligossacarídeos, amido e inulina (SANTANA; CARDOSO, 2008). Dentre os seus componentes se destaca a inulina, que possui alto poder adoçante e baixo valor calórico, possuindo ainda compostos fenólicos, proteínas, lipídeos, vitaminas e minerais. Os principais carboidratos presentes são os frutooligossacarídeos, açúcares que não podem ser digeridos diretamente pelo organismo devido à ausência de enzimas necessárias para o seu metabolismo, sendo considerados compostos bioativos na alimentação humana (CASTILLO ALFARO; VIDAL MELGAREJO, 2005).

A vida de prateleira da batata yacon é muito curta devido, principalmente, ao seu alto teor de umidade (83-90\%) que o torna muito perecível mesmo sob refrigeração, constituindo um problema para seus distribuidores e consumidores. Após a colheita, são iniciadas transformações químicas e bioquímicas tais como a hidrólise dos frutooligossacarídeos em açúcares simples, reduzindo assim a funcionalidade deste tubérculo. Assim, a indústria procura continuamente por processos simples que visem preservar a qualidade nutricional, farmacêutica e sensorial a fim de proporcionar maior vida útil para a batata yacon (SANTANA; CARDOSO, 2008). Como é muito apreciada por suas características, e tendo um crescente valor de mercado, requer o emprego de técnicas apropriadas de processamento com a finalidade de aumentar a sua vida útil e disponibilizar um material durável para a comercialização, sendo a secagem um dos principais processos utilizados com essa finalidade.

A liofilização é um método de secagem que consiste numa técnica eficaz para a obtenção de produtos com tais características, podendo, no caso de polpas, obter-se um material em forma de pó, capaz de preservar essas características por longos períodos. Entretanto, produtos em pó de suco de frutas são geralmente muito higroscópicos e, por isso, geralmente se usa maltodextrina na sua formulação para reduzir esta característica.

Os principais fatores que influenciam durante o armazenamento de produtos em pó são o teor de água e a atividade de água, pois afetam diretamente as características e a estabilidade dos alimentos (SILVA et al., 2015). O comportamento higroscópico dos produtos é avaliado por meio das isotermas de sorção de umidade que são a representação gráfica do teor de umidade de equilíbrio em função da atividade de água em uma dada temperatura e fornecem informações para o desenvolvimento e otimização de processos, solução de problemas no armazenamento e para a predição do tempo de vida de prateleira (LIMA et al., 2008).

Produtos em pó são de fácil reconstituição e têm um mercado garantido, formado por consumidores em busca de alimentos de fácil preparo, principalmente se apresentarem o apelo de serem saudáveis e nutritivos. Este trabalho foi realizado com o objetivo de verificar o comportamento higroscópico do pó obtido pela liofilização do mix de batata yacon e suco de lima ácida Tahiti nas temperaturas de 20, 30 e $40{ }^{\circ} \mathrm{C}$.

\section{MATERIAL E MÉTODOS}

O trabalho foi realizado no Laboratório de Armazenamento e Processamento de Produtos Agrícolas -
LAPPA, da Unidade Acadêmica de Engenharia Agrícola da Universidade Federal de Campina Grande (UFCG).

As matérias-primas utilizadas foram: batata yacon (Smallanthus sonchifolia) e lima ácida Tahiti (Citrus latifolia). As batatas apresentavam bom estado de conservação e a lima ácida em estado de maturação maduros e as cascas com coloração esverdeada para amarelo, ambos oriundos do mercado local de Campina Grande - PB. A maltodextrina foi doação da Corn Products Brasil.

As batatas yacon e a lima ácida Tahiti foram submetidos a uma lavagem em água corrente e logo após, realizada a sanitização, imergindo-se os mesmos em recipiente contendo solução de hipoclorito de sódio com concentração de 50 ppm, durante 15 minutos, e por fim enxaguados em água corrente para retirar o excesso da solução de hipoclorito, em seguida foram expostos a temperatura ambiente para a secagem. $\mathrm{O}$ processamento iniciou-se pelas limas ácidas, que foram descascadas e partidas ao meio para a extração e obtenção do suco; as batatas foram descascadas manualmente com facas de aço inoxidável e cortadas em pequenos pedaços, imediatamente após o processamento, foram imersas no suco integral de lima ácida na proporção 2:1 (batata yacon: suco de lima), por um período de quinze minutos, com a finalidade de evitar a oxidação e inativar as enzimas. Em seguida, a mistura foi triturada/homogeneizada em liquidificador doméstico com adição de $10 \%$ de maltodextrina (DE $=20)$. A amostra foi congelada sobre as prateleiras de um freezer vertical durante 24 horas e submetida ao processo de liofilização em liofilizador, durante 48 horas. Após a liofilização, a amostra foi triturada em almofariz com pistilo, obtendo-se assim o mix em pó da batata yacon e suco de lima ácida (yacon + lima ácida + maltodextrina).

As isotermas de adsorção de umidade do mix em pó foram determinadas de acordo com a metodologia de Capriste e Rotstei (1982). As leituras das atividades de água (aw) do pó foram determinadas no higrômetro AquaLab 3TE (Decagon) nas temperaturas de 20,30 e $40{ }^{\circ} \mathrm{C}$. As amostras em triplicata, foram colocadas em recipientes de vidros herméticos contendo água destilada, e permaneceram nesses ambientes até atingirem o equilíbrio higroscópico.

$\mathrm{O}$ teor de umidade de equilíbrio em base seca (\% b.s.) foi calculado com base na diferença entre a massa da amostra no equilíbrio e a massa seca (Eq. 1), determinada em estufa a $105^{\circ} \mathrm{C}$ durante 24 horas.

$$
U_{e q}=\frac{m_{e q}-m_{s}}{m_{s}}
$$

Em que: Ueq - umidade de equilíbrio (\% base seca); meq - massa da amostra no equilíbrio; ms - massa seca da amostra $(\mathrm{g})$

Logo após o equilíbrio das amostras, as curvas da atividade de água em função da umidade de equilíbrio foram graficadas usadando os modelos de Peleg, GAB, Halsey, Oswin e Smith (Tabela 1), fazendo-se uso do programa Statistica, através de regressão não linear e dos métodos de estimação Quasi-Newton e Rosenbrock. 
Tabela 1. Modelos matemáticos utilizados para o ajuste das isotermas de adsorção do pó da batata yacon com suco de lima ácida Tahiti.

\begin{tabular}{cc}
\hline Modelo & Equação \\
\hline Peleg & $U_{e q}=k_{1} a_{w}{ }^{n_{1}}+k_{2} a_{w}{ }^{n_{2}}$ \\
Halsey & $\mathrm{U}_{\mathrm{eq}}=\frac{\mathrm{x}_{\mathrm{m}} \mathrm{Cka}_{\mathrm{w}}}{\left(1-\mathrm{ka}_{\mathrm{w}}\right)\left(1-\mathrm{ka}_{\mathrm{w}}+\mathrm{Cka}_{\mathrm{w}}\right)}$ \\
Oswin & $U_{e q}=\left(\frac{-a}{\ln \left(a_{w}\right)}\right)^{1 / b}$ \\
Smith & $\mathrm{U}_{\mathrm{eq}}=\mathrm{a}\left(\frac{\mathrm{a}_{\mathrm{w}}}{\left(1-\mathrm{a}_{\mathrm{w}}\right)}\right)^{\mathrm{b}}$ \\
\end{tabular}

Em que: $\mathrm{U}_{\mathrm{eq}}$ - umidade de equilíbrio; $\mathrm{X}_{\mathrm{m}}$ - umidade na monocamada molecular; $\mathrm{a}_{\mathrm{w}}$ - atividade de água, adimensional; $\mathrm{k}_{1}, \mathrm{k}_{2}, \mathrm{n}_{1}, \mathrm{n}_{2}, \mathrm{C}, \mathrm{K}, \mathrm{a}, \mathrm{b}$ - constantes das equações.

Os critérios usados para determinação do melhor modelo ajustado às isotermas de adsorção de umidade foram o coeficiente de determinação $\left(R^{2}\right)$ e o desvio percentual médio $(\mathrm{P})$, calculado conforme a Equação7:

$\mathrm{P}=\frac{100}{n} \cdot \sum_{i=1}^{n} \frac{\left|\left(\mathrm{M}_{\text {exp }}-\mathrm{M}_{\text {teor }}\right)\right|}{\mathrm{M}_{\exp }}$

em que: $\mathbf{M}_{\text {exp }}$ - valores obtidos experimentalmente; $\mathbf{M}_{\text {teor }}$ valores preditos pelo modelo; $\mathrm{n}^{-}$número de dados experimentais.

\section{RESULTADOS E DISCUSSÃO}

Na Tabela 2 observa-se os valores dos parâmetros dos modelos de Peleg, GAB, Halsey, Oswin e Smith, ajustados às isotermas de adsorção de umidade do pó da batata yacon com lima ácida Tahiti, os coeficientes de determinação $\left(\mathrm{R}^{2}\right)$ e os desvios percentuais médios (P), nas temperaturas de 20, 30 e $40{ }^{\circ} \mathrm{C}$. Os resultados obtidos foram verificados para as temperaturas estudadas, assim podendo serem utilizados para representar com boa precisão as isotermas de adsorção de umidade do pó da batata yacon com suco de lima ácida Tahiti dento da faixa de temperatura estudada.

Tabela 2. Parâmetros, coeficientes de determinação $\left(\mathrm{R}^{2}\right)$ e desvios percentuais médios $(\mathrm{P})$ dos modelos ajustados às isotermas de adsorção de umidade, a 20, 30 e $40^{\circ} \mathrm{C}$, do pó da batata yacon com suco de lima ácida Tahiti.

\begin{tabular}{|c|c|c|c|c|c|c|c|}
\hline \multirow{2}{*}{ Modelo } & \multirow{2}{*}{$\mathbf{T}\left({ }^{\circ} \mathbf{C}\right)$} & \multicolumn{4}{|c|}{ Parâmetros } & \multirow{2}{*}{$\mathbf{R}^{2}$} & \multirow{2}{*}{$\mathbf{P}(\%)$} \\
\hline & & $\mathbf{k}_{1}$ & $\mathrm{n}_{1}$ & $\mathbf{k}_{2}$ & $\mathbf{n}_{2}$ & & \\
\hline \multirow{3}{*}{ Peleg } & 20 & 33,0475 & 0,9520 & 159,3878 & 13,6240 & 0,9935 & 4,93 \\
\hline & 30 & 137,9924 & 12,1639 & 33,4997 & 1,1466 & 0,9980 & 3,47 \\
\hline & 40 & 29,5189 & 0,8972 & 122,2657 & 10,1940 & 0,9979 & 3,10 \\
\hline \multirow{4}{*}{ GAB } & $\mathbf{T}\left({ }^{\circ} \mathbf{C}\right)$ & $\mathbf{X}_{\mathrm{m}}$ & & & $\mathbf{k}$ & $\mathbf{R}^{2}$ & $\mathbf{P}(\%)$ \\
\hline & 20 & 7,6834 & & 5,5 & 0,9834 & 0,9869 & 6,02 \\
\hline & 30 & 7,6192 & & 42 & 0,9877 & 0,9955 & 4,52 \\
\hline & 40 & 7,9550 & & 38 & 0,9831 & 0,9962 & 3,77 \\
\hline \multirow{4}{*}{ Halsey } & $\mathbf{T}\left({ }^{\circ} \mathbf{C}\right)$ & \multicolumn{2}{|c|}{$\mathbf{a}$} & \multicolumn{2}{|c|}{ b } & $\mathbf{R}^{2}$ & $\mathbf{P}(\%)$ \\
\hline & 20 & \multicolumn{2}{|c|}{11,3647} & \multicolumn{2}{|c|}{$-0,7825$} & 0,9842 & 7,43 \\
\hline & 30 & \multicolumn{2}{|c|}{10,2713} & \multicolumn{2}{|c|}{$-0,8345$} & 0,9950 & 4,24 \\
\hline & 40 & \multicolumn{2}{|c|}{11,0559} & \multicolumn{2}{|c|}{$-0,8080$} & 0,9951 & 4,22 \\
\hline \multirow{4}{*}{ Oswin } & $\mathbf{T}\left({ }^{\circ} \mathbf{C}\right)$ & \multicolumn{2}{|c|}{$\mathbf{a}$} & \multicolumn{2}{|c|}{ b } & $\mathbf{R}^{2}$ & $\mathbf{P}(\%)$ \\
\hline & 20 & \multicolumn{2}{|c|}{14,6661} & \multicolumn{2}{|c|}{0,6794} & 0,9767 & 10,47 \\
\hline & 30 & \multicolumn{2}{|c|}{13,8404} & \multicolumn{2}{|c|}{0,7029} & 0,9903 & 6,87 \\
\hline & 40 & \multicolumn{2}{|c|}{14,3808} & \multicolumn{2}{|c|}{0,6973} & 0,9876 & 7,81 \\
\hline \multirow{4}{*}{ Smith } & $\mathbf{T}\left({ }^{\circ} \mathbf{C}\right)$ & \multicolumn{2}{|c|}{$\mathbf{a}$} & \multicolumn{2}{|c|}{$\mathbf{b}$} & $\mathbf{R}^{2}$ & $\mathbf{P}(\%)$ \\
\hline & 20 & \multicolumn{2}{|c|}{$-3,5729$} & \multicolumn{2}{|c|}{27,6716} & 0,9293 & 15,05 \\
\hline & 30 & \multicolumn{2}{|c|}{$-2,5414$} & \multicolumn{2}{|c|}{25,4619} & 0,9538 & 11,46 \\
\hline & 40 & \multicolumn{2}{|c|}{$-3,1786$} & \multicolumn{2}{|c|}{26,2495} & 0,9259 & 15,18 \\
\hline
\end{tabular}

Em que: T - temperatura

De acordo com os valores obtidos dos coeficientes de determinação $\left(\mathrm{R}^{2}\right)$ e dos desvios percentuais médios $(\mathrm{P})$, pode-se observar que os modelos de Peleg, GAB e Halsey apresentaram coeficientes de determinações maiores ou iguais a 0,98 e desvios percentuais médios menores que $10 \%$, podendo ser utilizados para representar com boa precisão as isotermas de adsorção de umidade do pó da batata yacon com lima ácida Tahiti. De acordo com Lomauro et al. (1985), o valor de $\mathrm{P}$ indica um bom ajuste quando abaixo de $10 \%$. O modelo de Smith, apesar de ter apresentado coeficientes de 
determinação entre $0,92-0,95$, resultou em desvios percentuais médios maiores que $11 \%$, devendo-se, portanto, evitar seu uso no ajuste das isotermas do pó da batata yacon com lima ácida Tahiti.

O modelo que melhor se ajustou aos dados experimentais foi o de Peleg, apresentando os maiores coeficientes de determinação $\left(\mathrm{R}^{2} \geq 0,9935\right)$ e os menores desvios percentuais médios $(\mathrm{P}<5)$. $\mathrm{O}$ modelo de Peleg também foi o que melhor se ajustou aos dados experimentais das isotermas de adsorção de produtos como: bioplástico à base de farinha de mandioca da variedade CM 7951-5, nas temperaturas de 15,25 e $35^{\circ} \mathrm{C}$, apresentando $\mathrm{R}^{2}>0,996$ e erro médio relativo menor ou igual a $10 \%$ (NAVIA et al., 2011) e na farinha da casca da manga, seca em estufa a $50{ }^{\circ} \mathrm{C}$, apresentando $\mathrm{R}^{2}>0,99$ e $\mathrm{P}<6,20 \%$ (FEITOSA et al., 2013).

Constata-se também para o modelo de Peleg que as constantes do modelo k1, n1, k2 e n2 não apresentaram nenhum tipo de tendência com o aumento da temperatura das isotermas. Contrariamente, Thys et al. (2010) verificaram para as isotermas de adsorção de umidade do amido de pinhão que, com o aumento da temperatura entre 20,30 e $40{ }^{\circ} \mathrm{C}$, houve um aumento do parâmetro k1 e uma redução de k2.

$\mathrm{O}$ modelo de GAB também se ajustou com precisão às isotermas de adsorção de umidade da manga em pó, nas temperaturas de 25,30 e $35^{\circ} \mathrm{C}$, obtida por liofilização, apresentando $\mathrm{R}^{2}$ entre 0,9986 a 0,9993 (MOREIRA et al., 2013) e para a isoterma de adsorção a $23{ }^{\circ} \mathrm{C}$ do pó de framboesa (Rubusidaeus), produzido por liofilização, apresentando coeficiente de determinação de 0,996 (SYAMALADEVI et al., 2009).

A umidade na monocamada $(\mathrm{Xm})$ do modelo de GAB indica o teor de umidade para uma armazenagem segura (MISHIRA et al., 1996). Corresponde à quantidade de água fortemente adsorvida, sendo considerado um valor crítico, acima do qual as taxas de algumas reações de degradação aumentam e a estabilidade diminui (COMUNIAN et al., 2011). Observa-se que o teor de umidade na monocamada molecular (Xm) apresentou valores muito próximos nas três temperaturas, variando entre 7,6192e 7,9550\% b.s. Kotovicz (2011). Ao avaliar o comportamento higroscópico da batata yacon submetida à secagem convectiva a $70{ }^{\circ} \mathrm{C}$ por 150 minutos, após pré-tratamento osmótico, obteve-se valor de Xm de $17,36 \%$, sendo este muito superior ao do pó de yacon com lima ácida Tahiti

Ainda com relação ao modelo de $\mathrm{GAB}$, constatou-se que, com o aumento da temperatura, a energia livre parcial molar, representada pelo parâmetro $\mathrm{C}$, apresentou um comportamento indefinido. $\mathrm{O}$ parâmetro $\mathrm{K}$ apresentou-se numa faixa de 0,98 para todas as temperaturas estudadas, ou seja, valores $<1$. Valor próximo foi relatado por Oliveira et al. (2014) para o pó liofilizado da polpa de cajá integral, com $\mathrm{k}=0,9965$, afirmando os autores que o valor da força de interação entre o vapor de água e a matriz sólida do pó da polpa de cajá diminuiu com a adição da maltodextrina. Syamaladevi et al. (2009) afirmaram que os valores de $\mathrm{k}$ geralmente variam entre 0,7 e 1 para materiais alimentícios. $\mathrm{O}$ pó da batata yacon com suco de lima ácida Tahiti, portanto, se inclui nessa faixa.
De acordo com a classificação de Blahovec (2004) as isotermas de adsorção de umidade do pó da batata yacon com suco de lima ácida Tahiti são do Tipo II, pois apresentaram valores de $0<\mathrm{K} \leq 1$ e $\mathrm{C}>2$. Para Brunauer et al. (1938) e Alcântara et al. (2009), a curva do tipo II apresenta forma sigmoidal e é observada principalmente em isotermas de produtos orgânicos. Curvas características do Tipo II abrangem uma série de produtos, os quais foram relatados por diversos autores como, por exemplo: Mosquera et al. (2012) para o morango em pó, obtido por liofilização, com e sem maltodextrina e goma arábica; Aguirre-Cruz et al. (2010) para a isoterma da farinha de banana na temperatura de $30{ }^{\circ} \mathrm{C}$ que apresentou forma sigmoidal; Melo et al. (2011) para as isotermas de adsorção de umidade, a $25^{\circ} \mathrm{C}$, da casca, polpa e parte fibrosa do buriti e Choudhury et al. (2011) para as isotermas de adsorção do broto de bambu com forma sigmoidal do tipo II. Classificação diferente foi verificada por Bernstein e Noreña (2014) para as isotermas de adsorção do yacon (Smallanthus sonchifolius) entre as temperaturas de 25 ${ }^{\circ} \mathrm{C}$ e $40{ }^{\circ} \mathrm{C}$, desidratado em ar quente a $50{ }^{\circ} \mathrm{C}$, como sendo do tipo III. Isotermas do tipo III são características de materiais ricos em carboidratos, como é o caso da batata yacon e, provavelmente, no presente trabalho a classificação foi diferente em razão da adição do suco de lima ácida Tahiti.

Comparando os valores das umidades na monocamada do modelo de GAB, apesar dos valores muito próximos, verifica-se um pequeno aumento entre as temperaturas de 30 e $40^{\circ} \mathrm{C}$. Segundo Ferreira e Pena (2003), este não é um comportamento comum e o aumento da temperatura pode ocasionar alterações na estrutura física do produto, disponibilizando um número maior de sítios ativos com afinidade por moléculas de água ou pode originar um aumento na solubilidade de solutos intrínsecos ao produto, fazendo com que um número maior de moléculas de água fique retido na monocamada.

Blahovec (2004) afirmou que os parâmetros do modelo de Oswin devem apresentar valores de "a" maiores que zero e de "b" menores que um. Estes parâmetros, dentro dos referidos intervalos, indicam que não há ponto de inflexão na curva, ou seja, não há mudanças na concavidade das funções e, logo, esses parâmetros possuem consistência matemática e física. Para o pó da batata yacon com suco de lima ácida Tahiti verifica-se que os parâmetros do modelo de Oswin resultaram em valores para "a" maiores que zero e "b" menores que um. Kotovicz (2011), ao estudar a higroscopicidade da batata yacon submetida à secagem convectiva a $70{ }^{\circ} \mathrm{C}$ por 150 minutos (yacon seco, após prétratamento osmótico), ao aplicarem os modelos de GAB, BET e Oswin, observaram que todos os modelos apresentaram bom ajuste, porém o modelo de Oswin se destacou com o menor erro relativo.

Na Figura 1 observa-se a representação gráfica das isotermas de adsorção de água do pó da batata yacon com suco de lima ácida Tahiti nas temperaturas de 20,30 e $40^{\circ} \mathrm{C}$, com ajuste pelo modelo de Peleg considerado o melhor modelo. Nota-se pouca influência do aumento de temperatura sobre o comportamento das isotermas e verifica-se um aumento da atividade de água no pó da batata yacon com suco de lima ácida Tahiti com o aumento da umidade de equilíbrio. 
Figura 1. Isotermas de adsorção de umidade do pó da batata yacon com suco de lima ácida Tahiti com ajustes pelo modelo de Peleg.

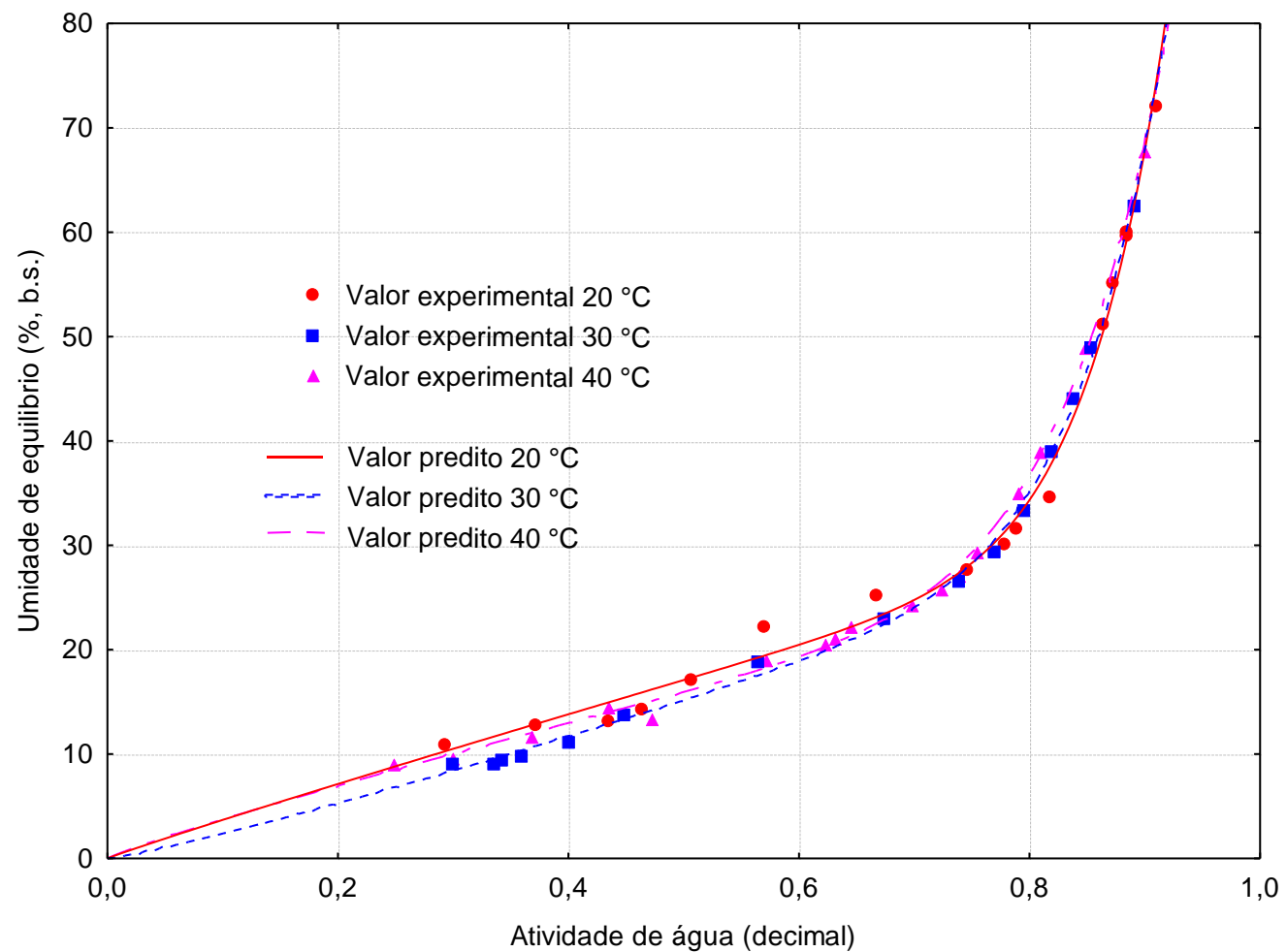

\section{CONCLUSÕES}

A temperatura de $30 \quad{ }^{\circ} \mathrm{C}$ mostrou menor higroscopicidade até a atividade de água em torno de 0,7 . O modelo que melhor se ajustou às isotermas de adsorção de umidade do pó da batata yacon com suco de lima ácida Tahiti foi o de Peleg, com as isotermas sendo classificadas como Tipo II. A umidade na monocamada molecular apresentou valor em torno de $7 \% \mathrm{~b}$.

\section{REFERÊNCIAS}

AGUIRRE-CRUZ, A.; ALVAREZ-CASTILLO, A.; CASTREJÓN-ROSALES， T.; CARMONA-GARCIÁ, R.; BELLO-PEREZ L, A. Moisture adsorption behavior of banana flours (Musa paradisiaca) unmodified and modified by acid-treatment. Starch-Starke, Weinheim, v.62, n.12, p.658-666, 2010.

ALCÂNTARA, S. R.; ALMEIDA, F. A. C.; SILVA, F. L. H.; GOMES, J. P. Isotermas de adsorção do pedúnculo seco do caju. Revista Brasileira de Engenharia Agrícola e Ambiental, Campina Grande, v.31, n.1, p.81-87, 2009.

BERNSTEIN, A.; NOREÑA, C. P. Z. Estudo da termodinâmica, estruturais e propriedades de qualidade de yacon (Smallanthus sonchifolius) durante a secagem. Food and Bioprocess Technology, Clevelãndia, v.7, n.1, p.148-160, 2014.

BLAHOVEC, J. 2004. Sorption isotherms in materials of biological origin mathematical and physical approach. Journal of Food Engineering, London, v.65, n.4, p.489-495, 2004.
BRUNAUER, S.; EMMETT, P. H.; TELLER, E. Adsorption of Gases in Multimolecular Layers. Journal of the American Chemical Society. v. 60, n. 2, p. 309-319, 1938.

CAPRISTE, G. H.; ROTSTEIN E. Prediction of sorptional equilibrium data for starchcontaining foodstuffs. Journal of Food Science, Oxford, v.47, n.5, p.1501-1507, 1982.

CASTILlO ALFARO, M. E.; VIDAL MELGAREJO, S. A. 2005. El yacón: unanuevaalternativaen la prevención y el tratamiento de la salud. Disponível em: <http://www.ilustrados.com/tema/6001/Yacon-nuevaalternativa-prevencion-tratamiento-salud.html>. Acesso em: 23 de fevereiro de 2017.

CHOUDHURY, D.; SAHU, J. K.; SHARMA, G. D. Moisture sorption isotherms, heat of sorption and properties of sorbed water of raw bamboo (Dendrocalamuslongispathus) shoots. Industrial Crops and Products. v.33, n.1, p.211-216, 2011.

COMUNIAN, T. A; MONTERREY-QUINTERO, E. S.; THOMAZINI, M.; BALIEIRO, J. C. C.; PICCONE, P.; PITTIA, P.; FAVARO-TRINDADE, C. S. Assessment of production efficiency, physicochemical properties and storage stability of spray-dried chlorophyllide, a natural food colourant, using gum Arabic, maltodextrin and soy protein isolate-based carrier systems. International Journal of Food Science and Technology, Oxford, v.46, n.6, p.1259-1265, 2011.

FEITOSA, R. M.; OLIVEIRA, E. N. A.; SANTOS D. C.; FIGUEIRÊDO, R. M. F.; QUEIROZ, A. J. M. Isotermas de adsorção de umidade da farinha da casca da manga. Higiene Alimentar, Mirandópolis, v.27, n.(especial), p.3780-3784, 2013. 
FERREIRA, C. D.; PENA R. S. Comportamento higroscópico da farinha de pupunha (Bactrisgasipaes). Ciência e Tecnologia de Alimentos, Campinas, v.23, n.2, p.251-255, 2003.

KOTOVICZ, V. Otimização da desidratação osmótica e secagem do yacon (polymnia sonchifolia). 2011. 90f. Dissertação (Mestrado em Tecnologia de Alimentos) Universidade Federal do Paraná, Curitiba. 2011.

LIMA, E. E.; SILVA, A. S.; FIGUEIRÊDO, R. M. F.; QUEIROZ, A. J. M. Estudo das isotermas e calor isostérico de adsorção da farinha da coroa de frade. Revista Brasileira de Produtos Agroindustriais, Campina Grande, v.10, n.2, p. 163-170, 2008.

LOMAURO, C. J.; BAKSHI, A. S.; LABUZA, T. P. Evaluation of food moisture sorption isotherm equations. Part I: fruit, vegetable and meat products. LebensmittelWissenschaft \& Techonologies, v.18, n.2, p.111-117, 1985.

MELO, W. S.; PENA, R. S.; RODRIGUES, A. M. C.; SILVA, L. H. M. Comportamento higroscópico do buriti (Mauritia flexuosa). Ciência e Tecnologia de Alimentos, Campinas, v.31, n.4, p.935-940, 2011.

MISHIRA, V. K.; ORAIKUL, B.; TEMELLI, F. Physical characterization and water sorpion of freeze dried dulsePalmariapalmata powder. Journal of Food Processing and Preservation, v.20, n.1, p.25-39, 1996.

MOREIRA, T. B.; ROCHA, É. M. F. F.; AFONSO, M. R. A.; COSTA, J. M. C. Comportamento das isotermas de adsorção do pó da polpa de manga liofilizada. Revista Brasileira de Engenharia Agrícola e Ambiental, Campina Grande, v.17, n.10, p.1093-1098, 2013.
MOSQUERA, L. H.; MORAGA, G.; MARTÍNEZNAVARRETE, N. Critical water activity and critical water content of freeze-dried strawberry powder as affected by maltodextrin and arabic gum. Food Research International, Canadá, v.47, n.2, p.201-206, 2012.

NAVIA, D. P.; AYALA, A. A.; VILLADA, H. S. Isotermas de adsorción de bioplásticos de harina de yucamoldeados por compresión. Biotecnologíaen El sector Agropecuario y agroindustrial, v.9, n.1, p.77-87, 2011.

OLIVEIRA, G. S.; COSTA, J. M. C.; AFONSO, M. R. A. Caracterização e comportamento higroscópico do pó da polpa de cajá liofilizada. Revista Brasileira de Engenharia Agrícola e Ambiental, Campina Grande, v.18, n.10, p.1059-1064, 2014.

SANTANA, I.; CARDOSO, M. H. Raiz tuberosa de yacon (Smallanthussonchifolius): potencialidade de cultivo, aspectos tecnológicos e nutricionais. Ciência Rural, Santa Maria, v.38, n.3, p. 898-905, 2008.

SILVA, R. N. G.; FIGUEIRÊDO, R. M. F.; QUEIROZ, A. J. M.; FEITOSO, R. M. Isotermas de adsorção de umidadedo umbu-cajá em pó. Revista Educação Agrícola Superior, Campina Grande, v.30, n.1, p.33-36, 2015.

SYAMALADEVI, R. M.; SABLANI, S. S.; TANG, J.; POWERS, J.; SWANSON, B. G. State diagram and water adsorption isotherm of raspberry (Rubusidaeus). Journal of Food Engineering, London, v.91, n.3, p.460-467, 2009.

THYS, R. C. S.; NORENA, C. P. Z.; MARCZAK, L. D. F.; AIRES, A. G.; OLIVERA, F. C. 2010. Adsorption isotherms of pinhão (Araucaria angustifolia seeds) starch and thermodynamic analysis. Journal of Food Engineering, London, v.100, n.3, p.468-473, 2010. 